'Departamento cirugía vascular, Facultad de Medicina. Pontificia Universidad Católica de Chile. Santiago, Chile.

Los autores declaran no tener conflictos de interés. Trabajo no recibió financiamiento.

Recibido el 6 de agosto de 2020 aceptado el 5 de octubre de 2020.

Correspondencia a:

J. Ignacio Torrealba:

Diagonal Paraguay 362. Santiago, Chile. jitorrealba@gmail.com

\section{Manejo endovascular de la isquemia crítica distal: análisis de una serie contemporánea}

\author{
J. IGNACIO TORREALBA ${ }^{1}, \mathrm{~J}_{\text {FRANCISCO VARGAS }}{ }^{1}$, \\ LEOPOLDO A. MARINÉ ${ }^{1}$, MICHEL P BERGOEING ${ }^{1}$, \\ RENATO A MERTENS ${ }^{1}$, FRANCISCO J. VALDÉS ${ }^{1}$
}

\section{Endovascular management of chronic limb ischemia. Experience in 48 procedures}

Background: Chronic limb ischemia can lead to high rates of limb loss and mortality. Open surgery is the gold standard for treatment of distal disease. Endovascular surgery should have less complications with similar outcomes. Aim: To report a cohort of patients with distal arterial disease treated with endovascular surgery at our institution. Material and Methods: Review of angioplasty records of patients undergoing distal lower extremity endovascular procedures between 2016 and 2019. Demographics, comorbidities, form of presentation, type of intervention, perioperative complications, and length of stay were analyzed. The primary outcomes were wound healing, reinterventions and freedom from major amputation. Secondary outcomes were overall survival and amputation-free survival. Results: Forty-eight limbs of 41 patients with a mean age 75 years ( $78 \%$ males) were treated. Ninety-three percent had hypertension, $88 \%$ diabetes, $30 \%$ chronic kidney disease. $73 \%$ presented with major wounds. Plain balloon and drug coated balloon angioplasties were carried out in 65 and $31 \%$ of procedures respectively, with no difference in results. In $46 \%$ of the cases, only chronic total occlusions were treated. Wound healing was achieved in $85 \%$ of procedures and $90 \%$ of patients were free from amputation at one year of follow up. Complications were observed in $18 \%$ of procedures, perioperative mortality was $2 \%$ and one-year survival was $76 \%$. Conclusions: Endovascular therapy achieves high rates of wound healing and freedom from amputation with low perioperative mortality and moderate complication rates.

(Rev Med Chile 2020; 148: 1734-1741)

Key words: Angioplasty; Endovascular Procedures; Limb Salvage; Lower Extremity.

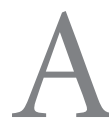
lrededor de $5 \%$ de la población adulta padece enfermedad arterial oclusiva de extremidades inferiores $(\mathrm{EAO})^{1}$, de éstos $1-3 \%$ se presentan inicialmente con isquemia crítica (presencia de dolor isquémico en reposo o heridas que no cicatrizan) y otro 5-10\% progresará a este estadio en un $a \tilde{n} \mathrm{O}^{2,3}$, con un elevado riesgo de pérdida de la extremidad sin tratamiento $(40 \%$ a un año $)^{1}$.
La EAO infragenicular presenta el grupo de mayor complejidad. Sumado a la alta tasa de comorbilidades y una baja expectativa de vida en este grupo, se agrega el riesgo de pérdida de la extremidad inferior, úlceras de difícil manejo y la necesidad de revascularización en un territorio arterial calcificado y con una pobre salida arterial hacia el pie (runoff).

La cirugía de revascularización mediante la 
construcción de un puente ha sido por décadas el tratamiento estándar, demandando destreza y experiencia para la realización de cirugía en arterias distales. Resultados del mundo real reflejan tasas subóptimas de permeabilidad, lo que se ve reflejado en altas tasas de amputación mayor ${ }^{4,5}$.

La cirugía endovascular ha intentado disminuir las tasas de complicaciones y la necesidad de hospitalización en unidades de alta complejidad, sin embargo, se ha cuestionado su baja permeabilidad $^{6}$ y eficacia, no habiendo en Chile datos claros de resultados.

Nuestro objetivo es mostrar una cohorte contemporánea de pacientes tratados por vía endovascular, evaluar nuestros resultados e identificar los factores asociados a la falla del tratamiento.

\section{Material y Método}

Se confeccionó una base de datos no identificable, respetando las normas éticas concordantes con la declaración de Helsinki.

El presente estudio fue aprobado por la unidad de ética y seguridad en investigación de la Pontificia Universidad Católica de Chile.

Se revisaron de forma retrospectiva todas las angioplastías de pacientes sometidos a intervención de vasos distales (arteria tibial anterior-ATA, tronco tibioperoneo-TTP, arteria tibial posterior-ATP, arteria peronea-AP) por ateromatosis entre los años 2016-2019. Se excluyeron los pacientes con angioplastías tibiales previas o para tratamiento de puentes quirúrgicos distales previamente confeccionados.

Se revisaron registros clínicos, electrónicos y angiografías, registrándose los datos epidemiológicos, presentación clínica, tratamiento, resultados y seguimiento incluyendo reintervenciones, cierre de heridas, amputación mayor y/o muerte.

Se evaluó la presencia de úlceras, su extensión y grado de infección según las clasificaciones WIfl ${ }^{7}$ y Rutherford.

La clasificación de WIfI evalúa la presencia de úlceras y su extensión, grado de infección e isquemia (Tabla 1).

No se evaluó el grado de isquemia según WIfI dada la falta de precisión del índice tobillo/brazo en enfermedad distal y la falta de datos de presión de ortejos en nuestra cohorte. La decisión de manejo endovascular quedó a decisión del tratante.

Nuestro grupo tiene una importante experiencia en el tratamiento con revascularización abier$\mathrm{ta}^{8}$, esta experiencia endovascular refleja solo $22 \%$ de todos los pacientes tratados por isquemia crítica con enfermedad infragenicular en el período.

Todos los casos fueron realizados en sala de angiografía o híbrida. El uso de ecografía para la punción arterial dependió del tratante, siendo siempre utilizado en accesos retrógrados distales (en arterias de la pierna o del pie) o en accesos

Tabla 1. Clasificación WIfI

\begin{tabular}{|lll|}
\hline Grado & Úlcera \\
\hline I & (menor) & Pequeña úlcera en pie, sin exposición ósea o sólo en falange \\
II & (mayor) & Úlcera con exposición ósea, gangrena ortejos \\
III & (extensa) & Úlcera profundacon exposición ósea, gangrena extensa \\
& & Infección \\
I & (leve) & Eritema local piel o tejido subcutáneo $<2 \mathrm{~cm}$ \\
II & (moderada) & Eritema con abceso u osteomielitis $>2 \mathrm{~cm}$ \\
III & (severa) & Respuesta inflamatoria sistémica $(\mathrm{SIRS})$ \\
& & Isquemia \\
I & (leve) & ITB* $0,6-0,79 ;$ presión ortejo $40-59 \mathrm{mmHg}$ \\
II & (moderada) & ITB $0,4-0,59 ;$ presión ortejo $30-39 \mathrm{mmHg}$ \\
III & (severa) & ITB $<0,4 ;$ presión ortejo $<30 \mathrm{mHg}$ \\
\hline
\end{tabular}

*Índice tobillo brazo. 
anterógrados. Se utilizó acceso retrógrado en casos donde la lesión no pudiera ser cruzada por vía anterógrada y siempre que el vaso distal estuviera permeable, tuviera diámetro de al menos 2 $\mathrm{mm}$ y estuviera libre de calcificaciones extensas. Se administró Heparina endovenosa en todos los casos una vez decidida la intervención.

Se realizó angioplastía con balones de 2 a 3,5 $\mathrm{mm}$ de diámetro. Según preferencia del tratante se utilizó angioplastía con balones cubiertos con Paclitaxel (DCB, drug coating balloon), o con balones simples (POBA, plain old balloon angioplasty).

En casos de disección limitante de flujo que no respondiera a inflados prolongados de balón, fue necesario instalar stents coronarios.

Todas las angiografías fueron revisadas, evaluándose el grado de estenosis u oclusión en las arterias tibial anterior, posterior, peronea o tronco tibioperoneo.

Se analizó el tratamiento efectuado y su resultado, clasificándolo en satisfactorio o no satisfactorio (persistencia de oclusión, estenosis $>30 \%$, en casos de angioplastía de más de un vaso se consideró satisfactorio si se obtuvo al menos un vaso permeable); se determinó el número de vasos permeables al final del procedimiento, el uso de DCB y su impacto en el cierre de heridas. En el caso del tronco tibioperoneo, se clasificó como una sola lesión si se localizaba en continuidad de lesión de las arterias tibial posterior o peronea adyacentes.

Se analizaron tasas de complicaciones mayores y menores perioperatorias.

Los objetivos primarios fueron de cierre de úlceras a 12 meses, tasa de reintervenciones y tasa de salvataje de extremidades.

Objetivos secundarios fueron analizar sobrevida general y sobrevida libre de amputación.

Se realizó análisis estadístico de variables continuas con U Mann-Whitney, de variables categóricas con test de Fisher. Se consideró como resultado estadísticamente significativo un $\mathrm{p}<0,05$.

Se confeccionaron curvas de Kaplan Maier para evaluar sobrevida global, sobrevida libre de amputación y salvataje de extremidades.

\section{Resultados}

En el período de estudio se realizaron 217 revascularizaciones distales de EEII por ateromatosis
(169 puentes y 48 angioplastías), se incluyen para esta revisión las 48 intervenciones tibiales efectuadas en 41 pacientes.

El seguimiento promedio fue de 282 días (18-820).

La edad promedio fue de 75 años (53-90 años), siendo $78 \%$ (32/41) hombres.

Las características demográficas y clínicas se detallan en la Tabla 2.

En $88 \%$ (42/48) de las intervenciones se realizó acceso femoral común contralateral. En 6\% (3/48) se realizó punción anterógrada por la arteria femoral común ipsilateral y en $6 \%$ se realizó punción retrógrada desde la arteria tibial anterior hacia proximal (Figura 1).

En 4\% (2/48) de las intervenciones se realizó angioplastía de arteria ilíaca ipsilateral y en $44 \%$ $(21 / 48)$ de arterias femoral superficial o poplítea.

\section{Tabla 2. Características demográficas y presentación clínica}

\begin{tabular}{|lr|}
\hline Comorbilidades & n (\%) \\
\hline Diabetes Mellitus & $35(85 \%)$ \\
\hline HTA & $37(90 \%)$ \\
\hline IRCr & $12(29 \%)$ \\
\hline Diálisis & $8(20 \%)$ \\
\hline Revascularización miocárdica & $10(24 \%)$ \\
\hline Tabaquismo & $10(24 \%)$ \\
\hline EAO con revascularización previa* & $8(20 \%)$ \\
\hline Lado izquierdo & $31(65 \%)$ \\
Lado derecho & $17(35 \%)$ \\
Presentación clínica por evento & \\
Claudicación invalidante & $4 \%(2)$ \\
Isquemia aguda & $4 \%(2)$ \\
Isquemia crítica & $92 \%(44)$ \\
Características heridas & \\
Infección & \\
WIfl 1 (leve) & \\
WIfl 2 (moderada) & \\
WIfl 3 (severa) & $19(40 \%)$ \\
WIfl 1 o mensión 2 o mayor (Rutherford V) & $18(38 \%)$ \\
\hline WIfl 3 o extensa (Rutherford VI) & \\
\hline
\end{tabular}

*Intervenciones excluidas en este análisis. 


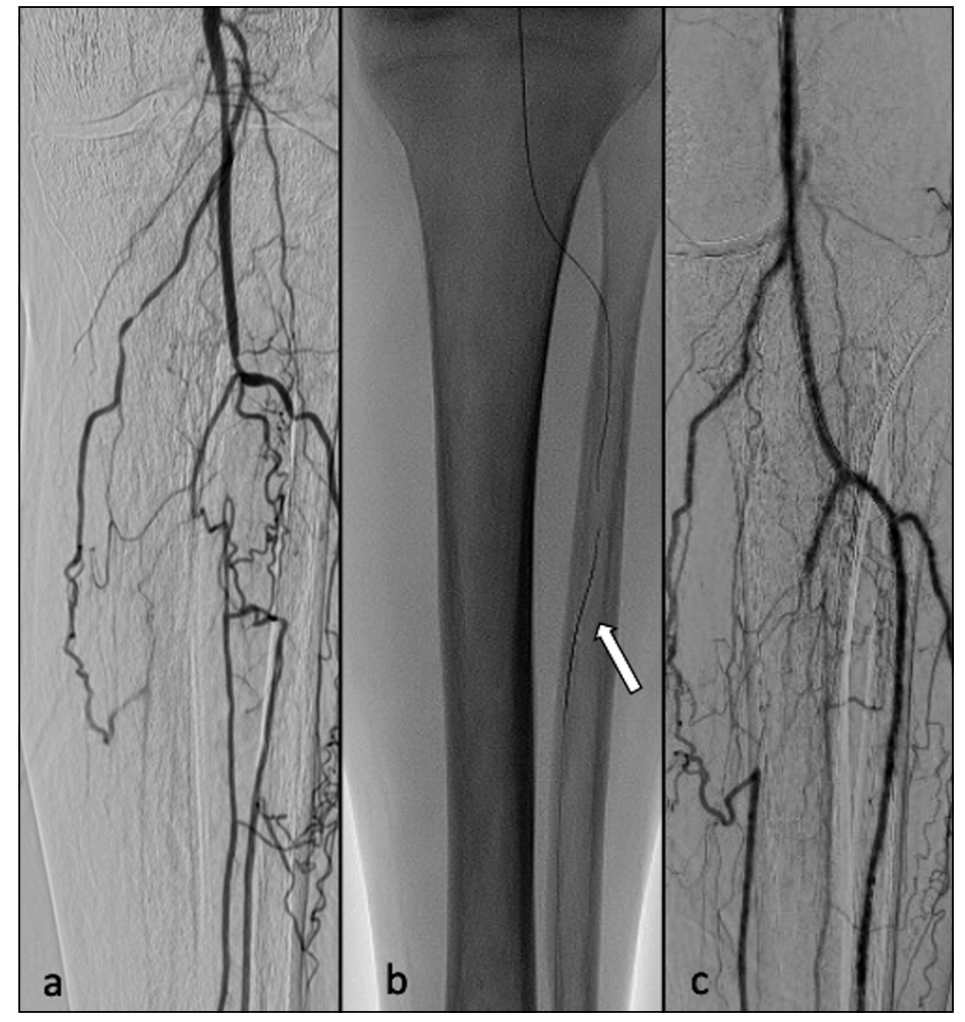

Figura 1. a) Angiografía demuestra oclusión de ATA, TTP, ATP y AP en su origen; b) Imposibilidad de penetrar oclusión de ATA, se introduce guía retrógrada desde acceso pedal (flecha blanca); c) Resultado exitoso luego de angioplastía con balón simple (POBA).
En 77\% (37/48) de los procedimientos se realizó angioplastía a un único vaso vs 11 casos $(23 \%)$ en que se realizó angioplastía a dos vasos, totalizando 59 vasos tratados.

La arteria más frecuentemente tratada fue la arteria tibial anterior (43\%). En la Tabla 3 se presenta la localización de las lesiones y vasos tratados.

En 50\% (24/48) de los procedimientos se realizó angioplastía de estenosis en un único vaso.

El 27\% (13/48) fueron recanalización exclusiva de la oclusión de un único vaso, con una extensión promedio de $5,2 \pm 3 \mathrm{~cm}$.

En 19\% (9/48) de las intervenciones hubo combinación de angioplastía de estenosis en un primer vaso asociado a recanalización de una oclusión en un segundo vaso y en $4 \%$ (2/48) hubo angioplastía de estenosis en dos vasos diferentes.

En quince casos (31\%) se realizó angioplastía con balón medicado (DCB) en 31 casos (65\%) se realizó angioplastía con balón simple (POBA) y dos casos $(4 \%)$ requirieron instalación adicional de stents.
Tabla 3. Localización y porcentaje de vasos tratados

\begin{tabular}{|c|c|c|}
\hline & $\begin{array}{c}\text { Presencia de lesiones } \\
\text { (n, \% total) }\end{array}$ & $\begin{array}{l}\text { PTA } 59 \text { vasos } \\
\text { (n, \% total) }\end{array}$ \\
\hline ATA & 48 (100\%) & 25 (43\%) \\
\hline ATP & 46 (96\%) & $12(20 \%)$ \\
\hline$A P$ & 38 (79\%) & $12(20 \%)$ \\
\hline TTP & $26 \quad(54 \%)$ & $10(17 \%)$ \\
\hline
\end{tabular}

El 100\% del grupo de PTA de 2 vasos se presentó con heridas en estadio WIFI 2 vs $61 \%$ en el grupo PTA de 1 vaso $(p=0,1)$. A su vez, el grupo de PTA de 2 vasos se presentó con un grado de infección al menos moderada en $72 \%$ de los casos vs $43 \%$ en el grupo de PTA de 1 vaso $(p>0,5)$.

El cierre de heridas se logró en $100 \%$ de pacientes con angioplastía a 2 vasos vs $83 \%$ en pacientes con angioplastía de un solo vaso $(\mathrm{p}=0,5)$. 
No hubo diferencias significativas $(p=0,5)$ entre pacientes con uso de DCB vs no DCB (POBA, stents) respecto al cierre de heridas.

Se obtuvo un resultado angiográfico satisfactorio en 32 casos (67\%). Sin embargo, la tasa de resultado satisfactorio post procedimiento no se asoció a una mejoría en cierre de heridas $(\mathrm{p}=0,4)$ ni a diferencias en la tasa de amputación mayor $(\mathrm{p}=0,5)$, pero sí a una menor tasa de complicaciones $(\mathrm{p}=0,04)$, relacionada principalmente a la conversión a cirugía abierta ( 3 casos).

El 54\% (26/48) de las extremidades requirió una amputación menor (ortejos o transmetatarsiana) debido a la presentación inicial. Un paciente (2\%) con infección severa (WIfI 3) requirió una amputación infracondílea a los 4 días post intervención fallida.

En 68\% (33/48) de la cohorte se logró estimar el cierre de heridas. En 7 extremidades no se puedo estimar el cierre de heridas dado que los pacientes murieron antes de los 12 meses. En cuatro extremidades se desconoce la evolución de las heridas por pérdida de seguimiento. Cuatro casos se presentaron originalmente sin heridas.

En 70\% (23/33) de los casos se logró el cierre de las heridas dentro de los primeros 6 meses post intervención y cinco (15\%) antes de completar los 12 meses, logrando una tasa de cierre de heridas de $85 \%(28 / 33)$.

En cinco casos no hubo cierre de heridas, requiriendo una amputación mayor. Se logró un salvataje de la extremidad de $92 \%$ y $85 \%$ a uno y dos años respectivamente (ES: 13\% ambos) (Figura 2).

Hubo 10\% (5/48) de reintervenciones alejadas, cuatro pacientes fueron reintervenidos por vía endovascular debido a reestenosis y un paciente tuvo una conversión a revascularización abierta, con un promedio de 3,6 meses $(0,1-6,5)$ desde la primera intervención.

El 10\% (5/48) de los procedimientos presentaron complicaciones perioperatorias menores (hematuria en dos casos, uno con hematoma de ingle pequeño, un delirium post procedimiento y una afasia espontáneamente recuperada sin evidencia de accidente cerebrovascular).

El 17\% (8/48) de las intervenciones presentaron complicaciones perioperatorias mayores: cinco casos con oclusión intraoperatoria de arterias distales, de los cuales 3 requirieron conversión a cirugía abierta, exitosa; dos pseudoaneurismas en el sitio de punción con tratamiento local y un paciente falleció por un síndrome coronario agudo a los 17 días post-procedimiento (totalizando 2\% de mortalidad perioperatoria).

El tiempo de estadía hospitalaria promedio fue de 9 días (1-40 días), determinado fundamentalmente por el tamaño e infección de las heridas al ingreso. En los casos de heridas menores (WIfI 1), la mediana de estadía fue significativamente menor que en el grupo de heridas mayores o extensas (WIfl 2 y 3 ), ( 1 vs 7 días, $\mathrm{p}=0,01$ ).

En nuestra serie hubo 41\% (17/41) de muertes durante el período de seguimiento.

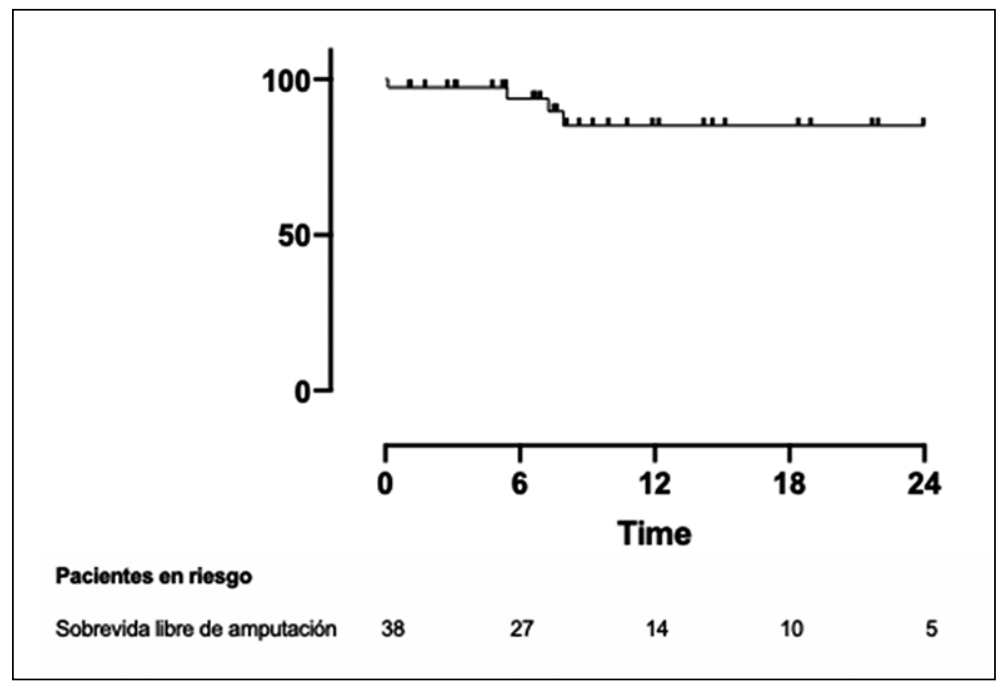

Figura 2. Curva Kaplan Meier para el salvataje de extremidad. 


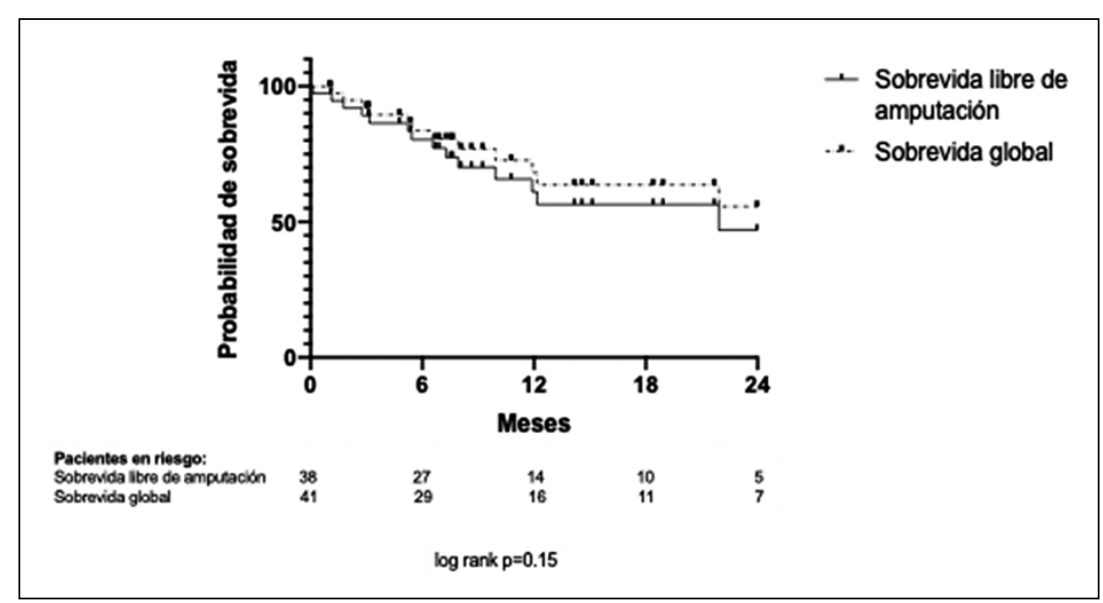

Figura 3. Curva de Kaplan Meier con sobrevida global y sobrevida libre de amputación.
Nueve (53\%) fallecieron por causa cardíaca, cuatro $(24 \%)$ por causa pulmonar, tres por sepsis abdominal y uno por hemorragia digestiva alta.

La curva de Kaplan-Meier muestra una sobrevida global de 76\% a los 12 meses (ES: 9\%) y $69 \%$ a los 2 años (ES: 11\%), con una mediana de sobrevida global de 25,8 meses y una mediana de sobrevida libre de amputación de 24,2 meses $(\mathrm{p}=0,62)$ (Figura 3).

\section{Discusión}

La revascularización de las extremidades inferiores es la única conducta que logra disminuir el riesgo de pérdida de extremidades en pacientes que se presentan con isquemia crítica.

Existe abundante literatura sobre el manejo de la enfermedad arterial distal mediante el uso de puentes venosos a arterias tibiales o del $\mathrm{pie}^{8-10}$. En centros de excelencia, se reportan tasas de permeabilidad y salvataje de extremidades cercanas a $80 \%$ y $90 \%$ a 5 años respectivamente ${ }^{8-10}$, sin embargo, para lograr dichos resultados deben combinarse varios factores como experiencia del equipo quirúrgico, apoyo capacitado de anestesiología y cuidados intensivos que no siempre están disponibles. Además, hay factores intrínsecos del paciente que pudieran afectar la revascularización tales como presencia de enfermedad arterial proximal, ausencia de una buena red de arterias distales a la enfermedad que lleguen hacia el pie (runoff) y particularmente la disponibilidad de un conducto autólogo adecuado, idealmente vena safena interna de al menos $3 \mathrm{~mm}$ de diámetro ${ }^{11}$. Las tasas de permeabilidad disminuyen considerablemente con conductos alternativos como venas del brazo o prótesis de PTFE ${ }^{12,13}$.

En las últimas dos décadas ha emergido la terapia endovascular para el manejo de la EAO de miembros inferiores. Actualmente, es el tratamiento de elección en la enfermedad aorto-ilíaca y femoral superficial, existiendo múltiples estudios que la posicionan como una alternativa eficaz a la cirugía convencional. Se han descrito y estudiado múltiples estrategias de manejo endovascular (POBA, stents, DCB, aterectomía $)^{14}$, logrando disminuir las complicaciones cardiovasculares y aquellas relacionadas con las heridas quirúrgicas ${ }^{15}$; aunque sacrificando permeabilidad a largo plazo $^{16}$.

En el manejo de la enfermedad distal por vía endovascular, no se ha observado el mismo desarrollo que en otros territorios y ha sido puesta en duda por su baja permeabilidad primaria, cercana a $50 \%$ a un año ${ }^{17,18}$.

En nuestra serie describimos una tasa de salvataje de extremidades por sobre $90 \%$ a un año, comparable con las mejores tasas de cirugía abierta ${ }^{8,10,11}$. El cierre de heridas se logró en 85\%, no logramos identificar factores significativos que pudieran afectar nuestro objetivo primario (uso de $\mathrm{DCB}$, número de vasos tratados), probablemente por el bajo poder de la muestra y una baja tasa de fracaso terapéutico.

Si bien la tasa de éxito del procedimiento (67\%) no es particularmente alta, cerca de $40 \%$ de las lesiones tratadas involucraron oclusiones com- 
pletas (y por lo tanto lesiones complejas, difíciles de tratar), lo que pone en evidencia la anatomía desfavorable de nuestros pacientes. Pese a ésto, logramos una adecuada tasa de cierre de heridas y salvataje de extremidad.

Hubo baja mortalidad perioperatoria (2\%), con una sobrevida promedio de 25 meses post intervención, lo que concuerda con la mortalidad reportada para este grupo de pacientes ${ }^{1,2}$.

Nuestra serie está conformada por pacientes con múltiples comorbilidades, sin embargo, hubo solo un caso de síndrome coronario agudo $(2 \%)$. Nuestra tasa de complicaciones mayores $(18 \%)$ si bien es alta, es similar a series de cirugía abierta $^{8,11}$, y está fuertemente influenciada por los casos en que hubo necesidad de conversión, con menor morbilidad cardiovascular o complicación de heridas que series de cirugía convencional ${ }^{8,10}$.

En todos los pacientes que requirieron conversión se logró realizar un puente venoso distal exitoso.

Si bien es cierto que aún con las técnicas endovasculares actuales la permeabilidad de los vasos tibiales es inferior a la reportada con la cirugía convencional, se debe considerar que este grupo de pacientes tiene una sobrevida limitada, por lo que no parece necesario obtener una permeabilidad a muy largo plazo dado que solamente se busca impedir la pérdida de la extremidad. Esto hace a la cirugía endovascular atractiva, logrando menores tasas de complicaciones cardiovasculares y un menor tiempo de estadía hospitalaria, sobretodo cuando la extensión de las heridas es pequeña (estadía promedio de sólo un día en esta serie).

Esta muestra de pacientes tratados por vía endovascular es sólo un quinto de la población tratada con revascularización en nuestro centro, por lo que los buenos resultados en cuanto a cierre de heridas y salvataje de extremidades no pueden ser extrapolados ampliamente a toda la población. Todos los pacientes debieran ser tratados en centros especializados donde se pueda ofrecer y realizar tanto revascularización abierta como endovascular dependiendo de las características particulares de cada caso. Se requiere, además, un seguimiento estrecho y capacidad de reintervención, abierta o endovascular en casos necesarios.

Este trabajo tiene varias limitaciones, el hecho de ser una revisión, sin un protocolo de selección clara de pacientes a manejo endovascular, con una variabilidad de tratamientos (POBA, DCB), lo hace susceptible de presentar sesgos de selección. No contamos con información completa para calcular permeabilidad, dado el manejo con seguimiento clínico y rara vez imagenológico de nuestros pacientes con angioplastías distales.

\section{Referencias}

1. Becker F, Robert-Ebadi H, Ricco JB, Setacci C, Cao P, de Donato G, et al. Chapter I: Definitions, epidemiology, clinical presentation and prognosis. Eur J Vasc Endovasc Surg. 2011; 42 Suppl 2: S4-12.

2. Norgren L, Hiatt WR, Dormandy JA, Nehler MR, Harris KA, Fowkes FG, et al; TASC II Working Group. Inter-Society Consensus for the Management of Peripheral Arterial Disease (TASC II). Eur J Vasc Endovasc Surg. 2007; 33 Suppl 1: S1-75.

3. Selvin E, Erlinger TP. Prevalence of and risk factors for peripheral arterial disease in the United States: results from the National Health and Nutrition Examination Survey, 1999-2000. Circulation 2004; 110: 738-43.

4. Brochado-Neto FC, Cury MV, Bonadiman SS, Matielo MF, Tiossi SR, Godoy MR, et al. Vein bypasses to branches of pedal arteries. J Vasc Surg 2012; 55: 746-52.

5. Gargiulo M, Giovanetti F, Bianchini Massoni C, Freyrie A, Faggioli G, Muccini N, et al. Bypass to the ankle and foot in the era of endovascular therapy of tibial disease. Results and factors influencing the outcome. J Cardiovasc Surg 2014; 55: 367-74.

6. Giles KA, Pomposelli FB, Spence TL, Hamdan AD, Blattman SB, Panossian H, et al. Infrapopliteal angioplasty for critical limb ischemia: relation of TransAtlantic InterSociety Consensus class to outcome in 176 limbs. J Vasc Surg 2008; 48: 128-36.

7. Mills JL Sr, Conte MS, Armstrong DG, Pomposelli FB, Schanzer A, Sidawy AN, et al; Society for Vascular Surgery Lower Extremity Guidelines Committee. The Society for Vascular Surgery Lower Extremity Threatened Limb Classification System: risk stratification based on wound, ischemia, and foot infection (WIfI). J Vasc Surg 2014; 59: 220-34.

8. Mertens R, Valdés F, Kramer A, Mariné L, Vergara J. Revascularización inframaleolar con vena safena: resultados a largo plazo. Arch Cir Vasc (Barcelona) 2000; 9: 211-21.

9. Pomposelli FB Jr, Marcaccio EJ, Gibbons GW, Campbell DR, Freeman DV, Burgess AM, et al. Dorsalis pedis arterial bypass: durable limb salvage for foot ischemia in patients with diabetes mellitus. J Vasc Surg 1995; 21 : 375-84. 
10. Schneider JR, Walsh DB, McDaniel MD, Zwolak RM, Besso SR, Cronenwett JL. Pedal Bypass versus Tibial Bypass with autogenous Vein: A Comparison of Outcome and Hemodynamic Results. J Vasc Surg 1993; 17: 1029-40.

11. Conte MS, Bandyk DF, Clowes AW, Moneta GL, Seely L, Lorenz TJ, et al. Results of PREVENT III: a multicenter, randomized trial of edifoligide for the prevention of vein graft failure in lower extremity bypass surgery. J Vasc Surg 2006; 43 (4): 742-51.

12. Sesto ME, Sullivan TM, Hertzer NR, Krajewski LP, O'Hara PJ, Beven EG. Cephalic vein grafts for lower extremity revascularization. J Vasc Surg 1992; 15: 543-9.

13. Parsons RE, Suggs WD, Veith FJ, Sánchez LA, Lyon RT, Marin ML, et al. Polytetrafluoroethylene bypasses to infrapopliteal arteries without cuffs or patches: a better option than amputation in patients without autologous vein. J Vasc Surg 1996; 23 (2): 347-54.

14. Rogers JH, Laird JR. Overview of new technologies for lower extremity revascularization. Circulation 2007; 116 (18): 2072-85.
15. Adam DJ, Beard JD, Cleveland T, Bell J, Bradbury AW, Forbes JF, et al; BASIL trial participants. Bypass versus angioplasty in severe ischaemia of the leg (BASIL): multicentre, randomised controlled trial. Lancet 2005; 366: 1925-34.

16. Bradbury AW, Adam DJ, Bell J, Forbes JF, Fowkes FG, Gillespie I, et al; BASIL trial Participants. Bypass versus Angioplasty in Severe Ischaemia of the Leg (BASIL) trial: An intention-to-treat analysis of amputation-free and overall survival in patients randomized to a bypass surgery-first or a balloon angioplasty-first revascularization strategy. J Vasc Surg 2010; 51 (5 Suppl): 5S-17S.

17. Giles KA, Pomposelli FB, Spence TL, Hamdan AD, Blattman SB, Panossian $\mathrm{H}$, et al. Infrapopliteal angioplasty for critical limb ischemia: relation of TransAtlantic InterSociety Consensus class to outcome in 176 limbs. J Vasc Surg 2008; 48 (1): 128-36.

18. Davies MG, El-Sayed HF. Outcomes of Isolated Tibial Endovascular Interventions for Tissue Loss in CLI Patients on Hemodialysis. J Endovasc Ther 2015; 22 (5): 681-9. 